\title{
Associação entre sintomas de DTM, bruxismo, estresse e fatores sociodemográficos em estudantes universitários
}

Association between TMD symptoms, bruxism, stress and sociodemographic factors in university students

Asociación entre síntomas de DTM, bruxismo, estrés y factores sociodemográficos en estudiantes universitarios

Recebido: 15/10/2021 | Revisado: 24/10/2021 | Aceito: 27/10/2021 | Publicado: 31/10/2021

\author{
Maurício Matheus \\ ORCID: https://orcid.org/0000-0003-1606-7886 \\ Centro Universitário da Fundação Hermínio Ometto, Brasil \\ E-mail: odontommatheus@yahoo.com.br \\ Heloísa Cristina Valdrighi \\ ORCID: https://orcid.org/0000-0001-7567-1990 \\ Centro Universitário da Fundação Hermínio Ometto, Brasil \\ E-mail: heloisavaldrighi@gmail.com \\ Mário Vedovello Filho \\ ORCID: https://orcid.org/0000-0002-5944-7937 \\ Centro Universitário da Fundação Hermínio Ometto, Brasil \\ E-mail: mario@vedovelloassociados.com.br \\ William Custódio \\ ORCID: https://orcid.org/0000-0003-1416-1414 \\ Centro Universitário da Fundação Hermínio Ometto, Brasil \\ E-mail: wcust@hotmail.com \\ Giovana Cherubini Venezian \\ ORCID: https://orcid.org/0000-0003-4643-7964 \\ Centro Universitário da Fundação Hermínio Ometto, Brasil \\ E-mail: giovanavenezian@fho.edu.br
}

\begin{abstract}
Resumo
O objetivo foi verificar a relação entre Sintomas de Disfunção Temporomandibular (DTM), bruxismo, estresse e fatores sociodemograficos em estudantes universitários. Foram aplicados questionários em 362 estudantes de Odontologia, na faixa etária de 17 a 56 anos de ambos os sexos. A coleta de dados foi realizada através do questionário para triagem para Dor Orofacial e DTM recomendado pela Academia Americana de Dor Orofacial, questionário de sintomas de Bruxismo baseado nos critérios da Academia Americana de Medicina do Sono, Escala de Estresse Percebido (PSS-14) e um questionário estruturado sociodemográfico. Os dados foram analisados por meio de regressão logística múltipla, considerando um nível de significância de 5\%. Os sintomas de DTM foram relatados por $60 \%$, o bruxismo por $37 \%$ e o estresse em $52 \%$ dos estudantes universitários avaliados. Verificou-se que universitários do sexo feminino, que estudam no período noturno e com estresse têm 3.36 (IC95\%: 1.87-6.01), 1.71 (IC95\%: 1.05-2.79) e 1.90 (IC95\%: 1.17-3.08) vezes mais chance, respectivamente, de apresentar pelo menos um sintoma de DTM $(P<0.05)$. Pessoas com um, dois e três ou mais sintomas de bruxismo têm 3.24 (IC95\%: 1.57-6.69), 4.62 (IC95\%: 1.91-11.16) e 18.75 (IC95\%: 4.39-80.13) vezes mais chance, respectivamente, de apresentar DTM do que as pessoas sem sintomas de bruxismo $(P<0.05)$. Conclui-se que houve associação entre a presença de sintomas de DTM, bruxismo e estresse.
\end{abstract}

Palavras-chave: Transtornos da Articulação Temporomandibular; Bruxismo; Estresse psicológico.

\begin{abstract}
The objective was to verify the relationship between Symptoms of Temporomandibular DIsorders (TMD), bruxism, stress, and sociodemographic factors in university students. Questionnaires were applied to 362 dentistry students, aged between 17 and 56 years of both sexes. Data collection was performed using the Orofacial Pain and TMD screening questionnaire recommended by the American Academy of Orofacial Pain, Bruxism symptoms questionnaire based on the American Academy of Sleep Medicine criteria, Perceived Stress Scale (PSS-14), and a structured sociodemographic questionnaire. Data were analyzed using multiple logistic regression, considering a significance level of $5 \%$. TMD symptoms were reported by $60 \%$, bruxism by $37 \%$, and stress by $52 \%$ of university students evaluated. It was found that female college students who study at night and with stress have 3.36 (95\%CI: 1.87-6.01), 1.71 (95\%CI: 1.05-2.79), and 1.90 (95\%CI: 1.17-3.08) times more chance, respectively, of having at least one TMD
\end{abstract}


symptom $(P<0.05)$. People with one, two, and three or more symptoms of bruxism are 3.24 (95\%CI: 1.57-6.69), 4.62 (95\%CI: 1.91-11.16), and 18.75 (95\%CI: 4.39-80.13) times more likely, respectively, to present TMD than people without bruxism symptoms $(P<0.05)$. It is concluded that there was an association between the presence of TMD symptoms, bruxism, and stress.

Keywords: Temporomandibular Joint Disorders; Bruxism; Psychological stress.

\section{Resumen}

El objetivo fue verificar la relación entre síntomas de Trastornos Temporomandibulares (DTM), bruxismo, estrés y factores sociodemográficos en estudiantes universitarios. Se aplicaron cuestionarios a 362 estudiantes de Odontología, con edades comprendidas entre los 17 y los 56 años de ambos sexos. La recolección de datos se realizó mediante el cuestionario de detección de dolor orofacial y DTM recomendado por la Academia Estadounidense de Dolor Orofacial, el cuestionario de síntomas de bruxismo basado en los criterios de la Academia Estadounidense de Medicina del Sueño, la Escala de Estrés Percibido (PSS-14) y un cuestionario sociodemográfico estructurado. Los datos se analizaron mediante regresión logística múltiple, considerando un nivel de significancia del 5\%. Los síntomas de DTM fueron reportados en un $60 \%$, bruxismo en un $37 \%$ y estrés en un $52 \%$ de los estudiantes universitarios evaluados. Se encontró que las estudiantes universitarias que estudian de noche y con estrés tienen 3.36 (IC95\%: 1.87 6.01), 1.71 (IC95\%: 1.05-2.79) y 1.90 (IC95\%: 1.17-3.08) veces más probabilidad, respectivamente, de tener al menos un síntoma de DTM $(P<0.05)$. Las personas con uno, dos y tres o más síntomas de bruxismo tienen 3.24 (IC95\%: 1.57-6.69), 4.62 (IC95\%: 1.91-11.16) y 18.75 (IC95\%: 4.39-80.13) veces más probable, respectivamente, para presentar DTM que las personas sin síntomas de bruxismo $(P<0.05)$. Se concluye que hubo asociación entre la presencia de síntomas de DTM, bruxismo y estrés.

Palabras clave: Trastornos de la articulación temporomandibular; Bruxismo; Estrés psicológico.

\section{Introdução}

Segundo a Academia Americana de Dor Orofacial, as Desordens Temporomandibulares (DTM) são definidas como um conjunto de distúrbios que envolvem os músculos mastigatórios, a articulação temporomandibular e estruturas associadas (Greene, Klasser \& Epstein, 2010). A DTM é uma das principais causas de dor não dental na região orofacial, impactando negativamente na qualidade de vida destes doentes (De Leeuw, 2008). Estudos epidemiológicos estimam que 40\% da população apresentem ao menos um sinal de DTM, como ruídos na ATM, e 33\% pelo menos um sintoma, como dor na face ou na ATM (De Leeuw, 2010; Klasser, 2015).

A etiologia da DTM é considerada multifatorial. Variações anatômicas no sistema mastigatório, dores em outras regiões do corpo, fatores demográficos e características psicossociais tem sido mais tradicionalmente sido identificados. Fatores genéticos e as relações oclusais também têm sido citadas (Okeson, 2013). Além disso, relação entre sintomas de DTM e estresse já foi proposta por alguns pesquisadores (Augusto et al., 2016; Su et al., 2017). Sendo que, estudos verificaram que o tratamento do estresse emocional melhora o quadro clínico de DTM (Wexlwe \& Steed, 1998).

O estresse pode ser definido como sendo a maneira a qual o organismo responde a um estímulo, preparando o corpo para fugir ou lutar. O principal hormônio liberado durante um episódio estressante é o cortisol. Mudanças na sua secreção são observadas associadas ao estresse psicológico (Taylor et al., 2018). Sugere-se que o indivíduo que está sobre efeito do estresse, apresenta um desequilíbrio do sistema mastigatório e uma hiperatividade muscular prolongada, o que pode resultar em fadiga, desconforto e dor da musculatura envolvida (Wexlwe \& Steed, 1998).

Além disso, acredita-se que a ocorrência de bruxismo também está associada à fatores psicológicos. Atualmente, o bruxismo é definido atualmente como uma atividade repetitiva da musculatura mastigatória caracterizada pelo apertamento ou ranger de dentes e/ou por segurar ou empurrar a mandíbula (Lobbezoo et al., 2013). Entretanto, evidências recentes sugerem que fatores biológicos e exógenos também podem estar envolvidos na etiologia deste hábito parafuncional (Manfredini et al., 2017).

Apesar de vastamente estudada, a etiologia multifatorial da DTM dificulta a correlação direta com uma das causas (Michelotti \& Iodice, 2010) tornando sua relação com o estresse e o bruxismo complexa. Assim, este estudo avaliou a associação dos sintomas de DTM, bruxismo e estresse, em estudantes do curso de Odontologia. 


\section{Metodologia}

Este estudo foi previamente aprovado pelo Comitê de Ética em Pesquisa com Seres Humanos (CAAE $\# 54235616.0 .0000 .5385$ ). Todos os participantes foram informados sobre os procedimentos do exame e garantidos acerca do sigilo das informações coletadas.

\section{Delineamento do estudo}

Estudo observacional transversal foi realizado com indivíduos adultos matriculados no curso de graduação de Odontologia, de ambos os sexos e com idade entre 17 e 46 anos. Foram excluídos aqueles que relataram história de cirurgias e/ou tumores da cabeça e pescoço e aqueles que preencheram o questionário de forma incompleta. Dos 621 alunos matriculados, participaram da pesquisa 362 estudantes.

\section{Descrição da amostra}

A amostra foi calculada utilizando dados de um estudo piloto com margem de erro de $10 \%$, perda amostral de $20 \%$ e nível de confiabilidade de $95 \%$. O tamanho da amostra de 362 voluntários proporcionou poder do teste de 0.80 , com um nível de significância de 5\%, para odds ratios acima de 2.0 nas análises das associações da presença de DTM com bruxismo e estresse (EpiInfo version 7.2.1.0, Centers for Disease Control and Prevention, Atlanta, U.S.A.).

\section{Instrumentos utilizados para a coleta de dados}

$\mathrm{O}$ processo de coleta de dados foi realizado na seguinte ordem: questões relacionadas às características sociodemográficas; Questionário de Triagem para Dor Orofacial e DTM; Questionário para avaliação de sintomas de bruxismo; Versão brasileira da Escala do Estresse Percebido. Os questionários foram aplicados em sala de aula e foram preenchidos individualmente por cada aluno.

\section{Características sociodemográficas}

As características sociodemográficas coletadas foram: nome, idade, sexo, estado civil, filhos e período e curso em que estuda.

\section{Questionário para Triagem de Dor Orofacial e DTM}

Os sintomas de DTM foram avaliados por meio de um questionário autoexplicativo para triagem de Dor Orofacial e DTM recomendado pela Academia Americana de Dor Orofacial (De Leew, 2010) e validado para a língua portuguesa (Franco-Micheloni et al., 2014). O questionário apresenta dez perguntas com respostas sim/não. Sete perguntas do questionário estão relacionadas aos sintomas de DTM: dor ou dificuldade para abrir a boca; travamento mandibular; dificuldade ou dor ao mastigar, falar ou movimentar a boca; ruídos nas articulações ao abrir a boca ou mastigar; sensação de rosto cansado duro ou tenso; dor perto dos ouvidos, dos lados da cabeça ou nas bochechas; dores de cabeça, pescoço ou dor nos dentes com frequência. As demais questões são sobre uma ocorrência de trauma, oclusão e realização do tipo de tratamento para DTM. O indivíduo foi considerado com a presença de sintomas de DTM quando respondeu afirmativamente para pelo menos um dos sete sintomas do questionário (Filho et al., 2020).

\section{Questionário para avaliação de sintomas de bruxismo}

O bruxismo do sono foi determinado com base nos critérios diagnósticos da Academia Americana de Medicina do Sono (2005) (Winocur et al., 2011). O questionário refere-se aos eventos ocorridos nos últimos 6 meses. Os entrevistados 
foram classificados com sintomas de bruxismo se sua resposta fosse positiva para a pergunta 1 e/ou a pergunta 2, além de pelo menos uma resposta positiva a um sintoma listado na pergunta 3 conforme (Winocur et al., 2011). Além disso, o número de sintomas relatados na questão três também foi avaliado.

\section{Questionário de Estresse Percebido}

Para mensurar o estresse percebido, utilizou-se a versão brasileira da Escala de Estresse Percebido (Dias et al., 2015). A escala pode ser utilizada em qualquer grupo etário, pois não contém perguntas específicas. Apresenta 14 questões com opções de respostas variando de 0 a 5 ( $0=$ nunca; $1=$ quase nunca; $2=$ às vezes; $3=$ quase sempre $4=$ =sempre), no qual o indivíduo determina o grau que cada situação a ele é estressante. As questões com conotação positiva (4, 5, 6, 7, 9, 10 e 13) têm sua pontuação somada invertida, da seguinte maneira, $0=4,1=3,2=2,3=1$ e $4=0$, as de sentido negativo são somadas diretamente. As somas das 14 questões dos escores devem variar entre 0 a 56 pontos (Luft et al., 2007; Dias et al., 2015), com os níveis de estresse classificados como: baixo quando a pontuação estava entre 0 a 14 pontos; moderado de 15 a 28 pontos; alto de 29 a 42 pontos e muito alto de 43 a 56 (Pozzebon et al., 2016).

\section{Análise estatística}

Inicialmente foram construídas tabelas de distribuição de frequências. A seguir foram ajustados modelos de regressão logística simples entre cada variável e a variável de desfecho (presença de DTM), estimando-se os odds ratios brutos com os respectivos intervalos de 95\% de confiança. Depois, as variáveis com $P<0.20$ nas análises individuais foram testadas em modelos de regressão logística múltipla, hierarquizados, permanecendo nos modelos as variáveis com $P \leq 0.05$. A introdução das variáveis nos modelos ocorreu do nível distal (variáveis sociodemográficas) para o proximal (estresse) e os ajustes analisados pelo AICC (Corrected Akaike information criterion). Com base nos modelos de regressão múltipla foram estimados os odds ratios ajustados com os respectivos intervalos de 95\% de confiança. As análises foram realizadas no programa e R ( $\mathrm{R}$ Core Team, 2017. R: A language and environment for statistical computing. R Foundation for Statistical Computing, Vienna, Austria).

\section{Resultados}

Os sintomas de dor Orofacial e DTM mais prevalentes foram "dor de cabeça, pescoço ou dor nos dentes com frequência" (42.5\%); "barulho perto dos ouvidos quando abre a boca ou mastiga" (31.2\%) e "rosto cansado, duro ou tenso" (29.8\%) (Tabela 1). 
Tabela 1. Característica da amostra em relação a presença de sintomas de dor Orofacial e DTM (Questionário de triagem recomendado pela academia americana de dor orofacial).

\begin{tabular}{|c|c|c|}
\hline Sintomas & Frequência(n) & Porcentagem $(\%)$ \\
\hline Dificuldade, dor ou ambas ao abrir aboca & 49 & $13.5 \%$ \\
\hline Boca já ficou "travada" ou seu queixo já "caiu" & 28 & $7.7 \%$ \\
\hline Dificuldade, dor ou ambas ao mastigar, falar ou movimentar a boca & 36 & $9.9 \%$ \\
\hline Barulho perto dos ouvidos quando abre a boca ou mastiga & 113 & $31.2 \%$ \\
\hline Rosto cansado, duro ou tenso & 108 & $29.8 \%$ \\
\hline Dor perto dos ouvidos, dos lados da cabeça ou nas bochechas & 87 & $24.0 \%$ \\
\hline Dor de cabeça, pescoço ou dor nos dentes com frequência & 154 & $42.5 \%$ \\
\hline Sofreu alguma pancada na cabeça, pescoço ou queixo & 7 & $1.9 \%$ \\
\hline Alteração recente na mordida & 35 & $9.7 \%$ \\
\hline $\begin{array}{l}\text { Recebeu tratamento prévio para dor no rosto ou outro problema na } \\
\text { região do ouvido }\end{array}$ & 15 & $4.1 \%$ \\
\hline
\end{tabular}

Fonte: Autores.

A Tabela 2 apresenta as características da amostra em relação ao número de sintomas de dor Orofacial e DTM. Observou-se que $59.7 \%$ dos indivíduos apresentaram algum sintoma de dor orofacial e DTM, sendo que $25.2 \%$ apresentaram três ou mais sintomas.

Tabela 2. Característica da amostra em relação ao número de sintomas de dor Orofacial e DTM (Questionário de triagem recomendado pela academia americana de dor orofacial).

\begin{tabular}{c|c|c}
\hline Número de Sintomas & Frequência(n) & Porcentagem(\%) \\
\hline Ausente & 146 & $40.3 \%$ \\
\hline Um & 77 & $21.3 \%$ \\
\hline Dois & 48 & $13.3 \%$ \\
\hline Três & 33 & $9.1 \%$ \\
\hline Quatro & 23 & $6.4 \%$ \\
\hline Cinco & 9 & $2.5 \%$ \\
\hline Seis & 16 & $4.4 \%$ \\
\hline Sete & 10 & $2.8 \%$ \\
\hline
\end{tabular}

Fonte: Autores.

Tabela 3 apresenta a análise da associação entre fatores sociodemográficos, nível de estresse emocional e sintomas de bruxismo. Universitários do sexo feminino, que estudam no período noturno e com estresse têm 3.36 (IC95\%:1.87-6.01), 1.71 (IC95\%:1.05-2.79) e 1.90 (IC95\%:1.17-3.08) vezes mais chance, respectivamente, de apresentar pelo menos um sintoma de DTM $(P<0.05)$. Pessoas com um, dois e três ou mais sintomas de bruxismo têm 3.24 (IC95\%:1.57-6.69), 4.62 (IC95\%:1.9111.16) e 18.75 (IC95\%:4.39-80.13) vezes mais chance, respectivamente, de apresentar DTM do que as pessoas sem sintomas de bruxismo $(P<0.05)$. 
Tabela 3. Resultado das análises bruta e ajustada da associação entre a presença de DTM e as variáveis sociodemográficas, número de sintomas de bruxismo e estresse.

\begin{tabular}{|c|c|c|c|c|c|c|c|c|}
\hline \multirow{2}{*}{ Variável } & \multirow{2}{*}{ Categoria } & \multirow{2}{*}{$\mathrm{N}\left({ }^{@ \%)}\right.$} & \multicolumn{2}{|c|}{ DTM } & \multirow{2}{*}{ *OR bruto $\left({ }^{\$} \mathrm{IC} 95 \%\right)$} & \multirow{2}{*}{ p-valor } & \multirow{2}{*}{$\begin{array}{c}\text { *OR ajustado } \\
\text { ('IC95\%) }\end{array}$} & \multirow{2}{*}{$\mathrm{p}$-valor } \\
\hline & & & $\begin{array}{c}\text { Não } \\
\mathrm{n}\left({ }^{\#} \%\right)\end{array}$ & $\begin{array}{l}{ }^{\&} \mathrm{Sim} \\
\mathrm{n}\left({ }^{\#} \%\right)\end{array}$ & & & & \\
\hline \multicolumn{9}{|l|}{ Nível distal } \\
\hline \multirow[t]{2}{*}{ Idade } & \multirow{2}{*}{$\begin{array}{l}\leq 21 \text { anos } \\
>21 \text { anos }\end{array}$} & $219(60.5 \%)$ & $92(42.0 \%)$ & $127(58.0 \%)$ & 1 & & & \\
\hline & & $143(39.5 \%)$ & $54(37.8 \%)$ & $89(62.2 \%)$ & $1.19(0.78-1.84)$ & 0.4209 & & \\
\hline \multirow{2}{*}{ Sexo } & Feminino & $283(78.2 \%)$ & $94(33.2 \%)$ & $189(66.8 \%)$ & $3.87(2.29-6.56)$ & $<0.0001$ & $3.36(1.87-6.01)$ & $<0.0001$ \\
\hline & Masculino & $79(21.8 \%)$ & $52(65.8 \%)$ & $27(34.2 \%)$ & 1 & & 1 & \\
\hline \multirow[t]{2}{*}{ Estado civil } & $\begin{array}{l}\text { Solteiro ou } \\
\text { divorciado }\end{array}$ & $301(83.1 \%)$ & $125(41.5 \%)$ & $176(58.5 \%)$ & 1 & & & \\
\hline & $\begin{array}{l}\text { Casado ou } \\
\text { união estável }\end{array}$ & $61(16.9 \%)$ & $21(34.4 \%)$ & $40(65.6 \%)$ & $1.35(0.76-2.41)$ & 0.3037 & & \\
\hline \multirow[t]{2}{*}{ Filhos } & Não & $327(90.3 \%)$ & $133(40.7 \%)$ & $194(59.3 \%)$ & 1 & & & \\
\hline & Sim & $35(9.7 \%)$ & $13(37.1 \%)$ & $22(62.9 \%)$ & $1.16(0.56-2.38)$ & 0.6860 & & \\
\hline \multirow[t]{2}{*}{ Período } & Integral & $188(51.9 \%)$ & $84(44.7 \%)$ & $104(55.3 \%)$ & 1 & & 1 & \\
\hline & Noturno & $174(48.1 \%)$ & $62(35.6 \%)$ & $112(64.4 \%)$ & $1.46(0.96-2.23)$ & 0.0800 & $1.71(1.05-2.79)$ & 0.0303 \\
\hline \multicolumn{9}{|c|}{ Nível mesial } \\
\hline \multirow{4}{*}{$\begin{array}{l}\text { Sintomas } \\
\text { de } \\
\text { Bruxismo }\end{array}$} & Nenhum & $229(63.3 \%)$ & $122(53.3 \%)$ & $107(46.7 \%)$ & 1 & & 1 & \\
\hline & Um & $46(12.7 \%)$ & $15(32.6 \%)$ & $31(67.4 \%)$ & $2.36(1.21-4.60)$ & 0.0033 & $3.24(1.57-6.69)$ & 0.0186 \\
\hline & Dois & $41(11.3 \%)$ & $7(17.1 \%)$ & $34(82.9 \%)$ & $5.54(2.36-13.00)$ & $<0.0001$ & $4.62(1.91-11.16)$ & 0.0063 \\
\hline & Três ou mais & $46(12.7 \%)$ & $2(4.4 \%)$ & $44(95.7 \%)$ & $25.08(5.94-105.94)$ & $<0.0001$ & $18.75(4.39-80.13)$ & 0.0025 \\
\hline \multicolumn{9}{|c|}{ Nível proximal } \\
\hline \multirow[t]{2}{*}{ Estresse } & Médio/alto & $187(51.7 \%)$ & $56(29.9 \%)$ & $131(70.1 \%)$ & $2.18(1.61-3.81)$ & $<0.0001$ & $1.90(1.17-3.08)$ & 0.0092 \\
\hline & Ausente/baixo & $175(48.3 \%)$ & $90(51.4 \%)$ & $85(48.6 \%)$ & 1 & & 1 & \\
\hline
\end{tabular}

*Odds ratio. ${ }^{\$}$ Intervalo de confiança. ${ }^{\&}$ Categoria de referência; ${ }^{*}$ porcentagem nas linhas; ${ }^{\circledR}$ porcentagem nas colunas. Fonte: Autores.

\section{Discussão}

O presente estudo associou dos sintomas de DTM, bruxismo e estresse, em estudantes do curso de Odontologia. De acordo com os achados, a maior prevalência de sintomas relacionados à DTM foi observada em mulheres. Estes resultados confirmam estudos prévios que apontam uma maior prevalência no sexo feminino, em uma proporção de até 1:4, comprometendo com mais frequência uma faixa etária entre 20-40 anos de idade (Figueredo et al., 2009). Acredita-se que o nível de estrogênio pode favorecer distúrbios da ATM em mulheres, por afetar os processos de remodelação óssea, alterando a matriz extracelular e o volume do osso (Okuda et al., 1996). Outras hipóteses ainda relacionam a maior suscetibilidade de mulheres à influência das características fisiológicas, variações hormonais e estruturas do tecido conjuntivo e muscular, uma vez que a maior flacidez desses tecidos, relacionada com os níveis de estrogênio, explica o fato de esses tecidos apresentarem menor capacidade de suportar pressão funcional levando à DTM (Minghelli, Kiselova e Pereira, 2011). Para Medeiros et al., (2011), essa maior prevalência de DTM observada no sexo feminino também podem estar associadas ao fato das mulheres serem mais cuidadosas e atenciosas com a saúde que os homens e procurarem tratamento com maior frequência. Por fim, maior nível de estresse emocional também tem sido relacionado à presença de DTM em mulheres, devido ao maior estresse mental (Manfredi et al., 2006; Wahid et al., 2014).

Neste estudo, observou-se que os estudantes do período noturno têm mais chances de desenvolver DTM, fato que pode estar relacionado a dupla jornada de atividades relacionada a um maior número de tarefas, além da possibilidade de menos horas de sono, o que pode aumentar a chance de estresse emocional. O estresse exerce efeitos nos mediadores emocionais e biológicos, podendo ativar tendências predisponentes e desencadear o aparecimento de doenças. Níveis aumentados de estresse emocional podem trazer desequilíbrios e resultar em alterações neuro-endócrino-imunes (Cruz et al., 2008). 
Os achados mostraram ainda que ocorrência de sintomas de DTM esteve relacionada tanto com estresse como com sintomas de bruxismo, embora uma maior força de associação tenha sido encontrada com o bruxismo, porém acredita-se que o estresse possa determinar a ocorrência do hábito parafuncional, estando ambos, portanto, de forma associada, contribuindo para o surgimento e/ou manutenção da dor (Brandini et al., 2011). A hipótese de que o bruxismo está envolvido na predisposição, iniciação e perpetuação da DTM é mantida, principalmente, porque é alta a prevalência desse distúrbio do movimento em pacientes com DTM quando comparados à população em geral (Nagamatsu-Sakaguchi et al., 2008).

É importante ainda considerar na interpretação dos resultados deste estudo, que a coleta de dados foi realizada por meio de questionários direcionados à sintomatologia percebida, portanto utilizando uma avaliação subjetiva dos sintomas pelos participantes. Atualmente, o exame clínico associado à anamnese e até mesmo exames complementares são indicados para o diagnóstico da DTM, assim como a polissonografia é considerada o padrão-ouro para diagnóstico do bruxismo do sono, e uma avaliação por um psicólogo para confirmação do estresse emocional, porém, estudos polissonográficos, que requerem exames clínicos ou avaliação por profissionais especializados envolvem alto custo, difícil acesso e equipamentos específicos, o que impossibilita sua utilização em estudos populacionais, conforme já relatado por Fragoso et al., (2010).

Estudos epidemiológicos nesta área são importantes a fim de contribuir para o esclarecimento das complexas interrelações entre a DTM e seus fatores associados, e ao mesmo tempo esclarecer os profissionais da área e direcionar os indivíduos mais acometidos, visto que muitas vezes há necessidade de uma abordagem multiprofissional para que seja possível um tratamento mais completo e eficaz ao paciente.

De acordo com dados da Organização Mundial de Saúde (OMS), o estresse afeta mais de 90\% da população mundial e é considerada uma epidemia global, estando envolvido como fator que aumenta a predisposição a diversas doenças (Brandini et al., 2011).

\section{Conclusão}

Houve associação entre o sexo feminino, período noturno de estudo, relato de sintomas de bruxismo e estresse emocional percebido e a presença de sintomas de DTM.

\section{Referências}

Academia Americana de Medicina do Sono - AASM. (2005): ICSD-2 Classificação Internacional de Distúrbios do Sono: diagnóstico e manual de codificação. (2a ed.), Westchester: AASM.Bruxismo relacionado ao sono; 189-92.

Augusto, V. G., Perina, K. C. B., Penha, D. S. G., Santos, D. C. A. D., \& Oliveira, V. A. S. (2016): Temporomandibular dysfunction, stress and common mental disorder in university students. Acta Ortopédica Brasileira 24, 330-333.

Brandini, D. A., Benson, J., Nicholas, M. K., Murray, G. M., \& Peck, C. C. (2011): Chewing in temporomandibular disorder patients: an exploratory study of an association with some psychological variables. Journal of Orofacial Pain 25(1), 56-67.

Cruz, M. C. F. N., Braga, V. A. S., Garcia, J. G. F., Lopes, F. F., \& Maia, E. C. S. (2008): Condições bucais relacionadas como estresse: uma revisão dos achados atuais. Revista da Faculdade de Odontologia de Porto Alegre 49, 8-11.

De Leeuw, R. (2008): Orofacial Pain: Guidelines for Assessment, Diagnoses and management. Quintessence.

De Leeuw, R. (2010): Dor orofacial: guia de avaliação, diagnóstico e tratamento. (4a ed.), Quintessence; 2010.

Dias, J. C. R., Silva, W. R., Moroco, J., \& Campos, J. A. D. B. (2015): Escala de estresse percebido aplicada a estudantes universitárias: estudo de validação. Psychology, Community \& Health 4,1-13.

Figueiredo, V. M. G., Cavalcanti, A. L., Farias, A. B. L., \& Nascimento, S. R. (2009): Prevalência de sinais, sintomas e fatores associados em portadores de disfunção temporomandibular. Acta Scientiarum. Health Science 31,159-63.

Filho, J. C., Vedovello, S., Venezian, G. C., Vedovello Filho, M., \& Degan, V. V. (2020). Women's oral health-related quality of life as a risk factor for TMD symptoms. A case-control study. Cranio: the journal of craniomandibular practice, 1-5.

Fragoso, Y. D., Alves, H. H. C., Garcia, S. O., \& Finkelsztejn, A. (2010): Prevalence of parafunctional habits and temporomandibular dysfunction symptoms in patients attending a tertiary headache clinic. Arquivos de Neuro-Psiquiatria 68, 377-80. 
Franco-Micheloni, A. L., Fernandes, G., Gonçalves, D. A., \& Camparis, C. M. (2014): Temporomandibular disorders among Brazilian adolescents: reliability and validity of a screening questionnaire. Journal of Applied Oral Science 22, 314-22.

Greene, C. S., Klasser, G. D., \& Epstein, J. B. (2010): Revision of the American Association of Dental Research's Science Information Statement about Temporomandibular Disorders. Journal (Canadian Dental Association) 76, a115.

Klasser, G. D., Rei, N., \& Lavigne, G. J. (2015). Sleep bruxism etiology: the evolution of a changing paradigm. Journal (Canadian Dental Association) 81 , f2.

Lobbezoo, F., Ahlberg, J., Glaros, A. G., Kato, T., Koyano, K., Lavigne, G. J., de Leeuw, R., Manfredini, D., Svensson, P., \& Winocur, E. (2013): Bruxism defined and graded: an international consensus. Journal of Oral Rehabilitation 40, 2-4.

Luft, C. D. B., Sanches, S. O., Mazo, G. Z., \& Andrade, A. Versão brasileira da Escala de Estresse Percebido: tradução e validação para idosos. Revista de Saúde Pública 41, 606-15.

Manfredi, A. P. S., Bortolleto, P. P. B., Silva, A. A., Araújo, I. E. M., Araújo, S., \& Vendite, L. L. (2006): Environmental stress and temporomandibular disorder (TMD) among members of a public university in Brazil. Brazilian Journal of Oral Science 5, 1074-8.

Manfredini, D., Serra-Negra, J., Carboncini, F., \& Lobbezoo, F. (2017): Current Concepts of Bruxism. The International Journal of Prosthodontics 30, 437438.

Minghelli, B., Kiselova, L., \& Pereira, C. (2011): Associação entre os sintomas da disfunção temporomadimbular com fator psicológicos e alterações na coluna cervical em alunos da Escola Superior de Saúde Jean Piaget do Algarve. Revista Portuguesa de Saúde Pública 20, 140-7.

Nagamatsu-Sakaguchi, C., Minakuchi, H., Clark, G. T., \& Kuboki, T. (2008): Relationship between the frequency of sleep bruxism and the prevalence of signs and symptoms of temporomandibular disorders in an adolescent population. The International journal of prosthodontics 21, 292-298.

Pozzebon, D., Piccin, C. F., Silva, A. N. T., \& Correâ, E. C. R. (2016): Relationship among perceived stress, anxiety, depression and craniocervical pain in nursing professionals under stress at work. Fisioterapia em Movimento 29, 377-385.

Okeson, J. P. (2013): Tratamento das desordens temporomandibulares e oclusão. (7a ed.), Mosby Elsevier.

Okuda, T., Yasuoka, T., Nakashima, M., \& Oka, N. (1996): The effect of ovariectomy on the temporomandibular joints of growing rats. Journal of Oral and Maxillofacial Surgery: Official Journal of The American Association of Oral and Maxillofacial Surgeons 54, 1201-1211.

Su, N., Lobbezoo, F., Vanwijk, A., Vanderheijden, G. J. M. G., \& Visscher, C. M. (2017): Associations of pain intensity and pain-related disability with psychological and socio-demographic factors in patients with temporomandibular disorders: a cross-sectional study at a specialised dental clinic. Journal of Oral Rehabilitation 44, 187-96.

Taylor, S. E., Klein, L. C., Lewis, B. P., Gruenewald, T. L., Gurung, R. A., \& Updegraff, J. A. (2000): Biobehavioral responses to stress in females: tend-andbefriend, not fight-or-flight. Psychological review 107, 411-429.

Wahid, S. N. N., Yusof, Y., \& Razak, M. R. (2014): Math Anxiety among Students in Higher Education Level. Social and Behavioral Sciences 123, $232-237$.

Wexler, G. B., \& Steed, P. A. (1998): Psychological factors and temporomandibular outcomes. Cranio: The Journal of Craniomandibular Practice 16, $72-77$.

Winocur, E., Uziel, N., Lisha, T., Goldsmith, C., \& Eli, I. (2011): Self-reported bruxism - associations with perceived stress, motivation for control, dental anxiety and gagging. Journal of Oral Rehabilitation 38, 3-11. 